\title{
La paraphrase comme lieu d'observation et de pratique de la dimension subjective et intersubjective du lexique et du discours
}

\section{The paraphrase as a means for the observation and practice of subjective and intersubjective dimensions in lexicon and discourse}

\author{
Lidia Lebas-Fraczak \\ Université Clermont Auvergne, Laboratoire de Recherche sur le Langage, Clermont-Ferrand \\ lidia.fraczak@uca.fr
}

\begin{abstract}
In the context of complete meaningful linguistic activities such as writing summaries, the paraphrase prompts reflection on the interaction between words, meaning and discourse, and thus helps develop the lexical component of discourse competence. Paraphrasing exercises are useful for both native and foreign language practice, especially for future translators, and lend students greater awareness of the shades of meaning distinguishing synonymic expressions in given contexts. We show that variations in meaning due to the use of synonyms and morpho-syntactical reformulations may be analyzed in terms of point of view and focalization, thereby taking into account the subjective and intersubjective dimensions of lexicon and discourse.
\end{abstract}

Keywords: paraphrase, lexical competence, semantic analysis, synonymy, focalization

\section{INTRODUCTION}

La paraphrase peut être définie comme une « relation entre formulations linguistiques » établie grâce à «l'activité de reformulation », celle-ci étant une «construction signifiante » (Fuchs, 1994, pp. 3, 25). Placée dans un cadre pédagogique mettant en œuvre des activités discursives complètes, telles que l'écriture de résumés de 
textes, la paraphrase permet de mener un travail sur l'interaction entre formes, sens et discours, en contribuant ainsi, en particulier, au développement du volet lexical de la compétence discursive, tout en permettant de mettre en évidence les liens entre le lexique et la cohésion textuelle (concernant la contiguïté sémantique) ainsi que, plus largement, la cohérence (consistant dans le rapport d'idées qui doivent s'accorder entre elles). Les exercices de paraphrase sont intéressants tant pour un public d'étudiants francophones que pour les étudiants pour qui le français est une langue étrangère et une matière de spécialité, notamment les futurs traducteurs et interprètes, leur permettant de se sensibiliser aux nuances de sens distinguant des expressions synonymiques dans des contextes particuliers. Nous essaierons de montrer que les variations de sens liées à l'emploi de synonymes, mais aussi aux reformulations (morpho-)syntaxiques, se laissent analyser en termes de point de vue ou de focalisation, ce qui revient à tenir compte de la dimension subjective et intersubjective de la langue et du discours.

Après avoir introduit des définitions et des postulats constituant le cadre théorique, nous proposerons un exemple d'application didactique, en illustrant le cheminement à partir de la lecture du texte source, en passant par l'écriture d'un résumé lexicalement fidèle, jusqu'au travail de production de paraphrases, cette dernière étape impliquant une analyse lexicale fondée sur un critère «attentionnel ». Comme nous le verrons, même si écrire et résumer sont a priori des tâches individuelles, les activités proposées se prêtent à un travail collectif et coopératif, avec tous les avantages que ce mode de travail implique.

\section{FACTEURS EN JEU DANS LA PARAPHRASE}

Comme le fait remarquer Fuchs, « les choix effectués par le sujet lors de la transformation du contenu sont évidemment fonction de l'ensemble des paramètres constitutifs de l'activité discursive », y compris « de l'objectif poursuivi par le sujet reformulant (selon le type de tâche langagière dans laquelle il est engagé) » et «selon la représentation qu'il a de la situation d'énonciation du T-source et de sa propre situation de reformulation » (1994, pp. 31-32). Bien que la reformulation visant une paraphrase soit donc fondée sur les mêmes paramètres que la production discursive « directe », elle peut donner lieu plus naturellement à une approche réflexive et métalinguistique, dans la mesure où, s'agissant de transformer un texte, le choix des expressions à employer se fera consciemment en fonction d'autres expressions, celles employées dans le texte d'origine et celles constituant des alternatives. Cette situation discursive est donc propice à la comparaison et à la différenciation d'expressions linguistiques, ce qui passe par la réflexion sur leurs caractéristiques sémantico-pragmatiques. 
La tâche de reformulation est comparable à celle de traduction (qui peut être vue comme une paraphrase «interlinguale»), l'une des différences étant que dans le cas de la paraphrase «intralinguale » (au sein donc d'une même langue), le but communicatif du sujet reformulant peut plus facilement diverger de celui de l'auteur du texte d'origine, ne serait-ce que par le fait de rechercher l'économie d'expression, comme dans le cas du résumé de texte. C'est notamment grâce à la nécessité de se pencher sur la visée communicative de l'auteur du texte original (la visée « globale » mais aussi les visées «locales») et, en cas de divergence, sur celle du sujet reformulant, que la reformulation en vue des paraphrases est une activité particulièrement intéressante pour mener un travail sur les liens entre formes, sens et discours, dans le but de développer la compétence discursive.

\section{DIMENSION SUBJECTIVE ET INTERSUBJECTIVE}

Nous allons nous concentrer en particulier sur les reformulations lexicales, en montrant que les différences entre des expressions synonymiques, partageant des caractéristiques référentielles, peuvent s'analyser en termes de point de vue ou de focalisation. Car, comme le précise Krieg-Planque (2012), « le lexique offre souvent au locuteur la possibilité de présenter le réel d'un certain point de vue, par différence avec d'autres points de vue possibles » (p. 90). Cela renvoie à «l'élément subjectif de la langue », pour lequel, comme l'a souligné le père de la sémantique, Bréal (1897), «il ne s'agit (...) pas d'un accessoire, d'une sorte de superfétation, mais au contraire d'une partie essentielle»du sens (p. 265). On peut également citer Bakhtine (1929[2006]) et sa critique des linguistes qui, préoccupés par « la construction d'un système linguistique abstrait », «en sont arrivés à séparer l'appréciatif du significatif, et à considérer l'appréciatif comme élément marginal de la signification » (p. 150). Il est important de faire remarquer, cependant, que la subjectivité dans la langue ne concerne pas uniquement la manifestation d'une attitude «appréciative » par rapport à un référent, car, comme le précise Kerbrat-Orecchioni (2009), «tous ses choix impliquent le locuteur » (p. 36) et donc sa subjectivité.

Il est également important de prendre en compte le fait que le point de vue du locuteur passe par le choix de focaliser tel ou tel aspect du référent, ou de ce à quoi le discours ou l'énoncé fait référence. En effet, comme le fait remarquer Gardiner (1932[1989]), "même si l'on admet que le discours peut, éventuellement, faire référence à des choses concrètes, on sera au moins obligés de préciser que c'est toujours un aspect particulier de ces choses qui est évoqué » (p. 28) ${ }^{1}$. Comme le précise Le Ny (2005), «le processus de construction du sens comporte une focalisa-

\footnotetext{
${ }^{1}$ L'auteur précise qu' «il est préférable de décrire ces aspects comme des 'pensées' ou des 'pensées sur les choses' que comme de véritables 'choses' » (ibid.).
} 
tion, une mise en jeu de l'attention interne, sur certaines des parties de la représentation $\gg$ (p. 368).

Cependant, en prenant en compte la focalisation (d'un aspect d'une «chose » ou «représentation»), il convient de parler d'intersubjectivité plutôt que de subjectivité, dans la mesure où le choix d'un aspect à focaliser implique non seulement le «sujet parlant» (locuteur) mais aussi le «sujet écoutant» (auditeur ou interlocuteur), dans la mesure où ce choix relève du «désir évident du locuteur d'amener habilement l'auditeur à diriger son attention dans une direction donnée » (Gardiner, 1932[1989], p. 72). Le principe d'attention partagée, impliquant pour le locuteur la nécessité de se préoccuper de l'interprétation de l'interlocuteur, permettant la construction d'un «monde intersubjectif », est désormais largement reconnu par les chercheurs en sciences cognitives comme étant fondamental pour la cognition et pour la communication humaines (cf. Tomasello, 2014). Le but communicatif du locuteur ne consiste donc pas seulement à exprimer une «perception » mais surtout à y associer un interlocuteur, comme le précise Gardiner (1932[1989]) dans la citation ci-dessous :

En fait, pour qu'il y ait discours, deux circonstances déterminantes doivent être réunies :

(1) la perception de quelque chose présentant un intérêt suffisant pour inciter à l'action et

(2) le désir d'associer à cette perception quelqu'un d'autre, d'une façon ou d'une autre. (pp. 62-63).

À l'idée selon laquelle le choix d'une expression oriente l'attention de l'interlocuteur sur un ou des aspects particuliers de la référence, il faut ajouter l'idée que ce choix lexico-pragmatique se fait en cohérence avec l'orientation communicative/argumentative de l'énoncé et du discours, celle-ci étant fonction du but communicatif poursuivi par le locuteur : le but «local» de l'énoncé et le but «global»du discours. Ainsi, il nous semble important de respecter, lors de l'analyse, «l'ordre méthodologique » préconisé par Bakhtine (1929[2006]) :

1. Les formes et les types d'interaction verbale en liaison avec les conditions concrètes où celle-ci se réalise.

2. Les formes des énonciations distinctes, des actes de parole isolés, en liaison étroite avec l'interaction dont ils constituent les éléments (...).

3. À partir de là, examen des formes de la langue dans leur interprétation linguistique habituelle (p. 137).

On peut faire le lien avec l'approche de Rastier (1987) et des linguistes pour qui une étude sémantique ne peut s'effectuer que par rapport à des contextes réels et précis, c'est-à-dire au sein de la «parole », ou du discours, et non pas au sein de la «langue».

La façon dont le sens, ou le «motif », d'un mot est «profilé » par son entourage contextuel a été illustrée par Longhi (2008) dans son analyse du terme intermittent, le profilage pouvant consister dans le fait de privilégier (donc de focaliser) une composante sémantique du motif au détriment d'une autre ; par exemple, la composante 
/métier/ plutôt que /statut/ dans un syntagme comme carte professionnelle d'intermittent (p. 116).

Ainsi, dans nos exercices, le choix du synonyme doit, pour être acceptable, se faire en fonction du sens de l'expression d'origine tel qu'il est construit dans le discours.

En tenant compte des arguments suivants de Galisson (1970:12), on peut préférer au terme «synonyme » celui de « parasynonyme » :

Pour que deux mots soient synonymes, il faudrait qu'ils aient le même sens et qu'ils soient toujours commutables. Or l'analyse sémique montre que deux mots ont rarement le même sens, et s'ils ont le même sens (...), l'analyse distributionnelle prouve qu'ils ne sont pas synonymes pour autant, puisqu'ils ne peuvent pas toujours commuter.

\section{LE PREMIER CONTEXTE PÉDAGOGIQUE : RESUMÉ EN FRANÇAIS LANGUE MATERNELLE}

Le cadre général des exercices de paraphrase, proposés à nos étudiants de première année de licence des Sciences du langage à l'université de Clermont-Ferrand, dans une partie relative au texte du cours intitulé «Langue, orthographe et texte», est celui du résumé de texte. L'intérêt théorique du résumé est que cette pratique discursive permet d'illustrer concrètement, à partir de productions des étudiants et des imperfections observées dans ces productions puis analysées collectivement, certaines caractéristiques du texte, concernant la cohérence et la cohésion. L'intérêt pratique de l'exercice de résumé dans un cours universitaire en France réside, entre autres, dans le fait que ce type de tâche est souvent proposé aux différents concours visés par les étudiants (ex. concours d'orthophonie, concours de professorat des écoles, concours administratifs), où il faut se plier à certaines règles, comme celles, citées ci-dessous, fournies par Le Bulletin Officiel qui, bien que datant de 1983, constituent toujours la référence des supports visant la préparation aux concours :

Le résumé suit le fil du développement. Il donne au texte, dans le même ordre, une version condensée mais fidèle. Il ne change pas le système de l'énonciation; il reformule le discours du texte initial sans prendre de distance. Il s'interdit un montage de citations : le candidat exprime dans son propre langage les assertions du texte. Il peut cependant, lorsqu'il s'agit de mots clés qu'il serait absurde de remplacer par de mauvais équivalents, reprendre les mots du texte et, par exception, citer entre guillemets une formule particulièrement significative. (B.O., juillet 1983)

On s'aperçoit, en lisant cette définition du résumé, et encore plus en essayent de la mettre en pratique, que l'exigence de «ne pas prendre de distance » (donc, de ne pas introduire sa subjectivité propre) et celle de s'exprimer «dans son propre langage » sont en réalité contradictoires, et semblent indiquer que les auteurs de ces prescriptions considèrent que la reformulation, ou recours à des synonymes, peut ne 
modifier que la forme du texte, puisque le texte reformulé est censé rester fidèle au texte d'origine et donc à son orientation attentionnelle et argumentative. Il s'agit donc pour les candidats, et, avant cela, pour les étudiants qui s'exercent à la rédaction de résumés, de trouver des reformulations ou des synonymes les moins divergents possible des formulations originales du point de vue communicatif et argumentatif, ce qui s'avère une tâche délicate, tout à fait comparable à celle d'un traducteur qui doit reformuler un texte dans une autre langue en essayant d'éviter le plus possible de s'en éloigner, pour respecter au mieux l'intention communicative de l'auteur. Cependant, on peut considérer, comme nous l'avons fait dans notre cours, que ces recommandations contraignantes et difficilement conciliables créent en même temps une situation favorable à une réflexion poussée sur les valeurs des différentes formes et expressions en lien avec le contexte, et donc à un travail enrichissant sur le lexique, le texte et le discours.

\subsection{CHOIX DE TEXTES ET RÉSUMÉS}

Les textes choisis pour les résumés sont assez courts (plus courts que ceux habituellement proposés aux concours), comme celui ci-dessous, qui nous servira de base pour tous nos exemples, ceux d'abord relatifs aux difficultés des étudiants, et ceux ensuite illustrant le travail autour de la paraphrase. Le texte en question s'intitule «Les illusions de la perception en politique » et est issu de le revue Philosophie magazine, $n^{\circ} 85$ (décembre 2014/janvier 2015).

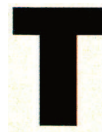
oute conscience est conscience perceptive, même laconscience de nous-mêmes", écrivait Maurice Merleau-Ponty dans Le Primat de la perception etses conséquences philosophiques (Verdier, 2014). Cela est également vrai en politique où les illusions de la perception quiretenaientl'attention du phénoménologue produisent d'étranges sortilèges, comme le montre l'enquête "Les périls de la perception ", menée par l'institut Ipsos dans quatorze pays et sur plus de 10000 personnes âgées de 16 à 64 ans. L'idée, très philosophique, est d'interroger les personnes non sur leurs opinions, mais sur leur perception de la société dans laquelle elles vivent, en mesurant l'écart entre le réel et l'image qu'elles s'en font.

Sur une série d'enjeux - nombre de chômeurs ou d'immigrés, place de l'islam par rapport au christianisme, vieillissement de la population -, les peuples n'ont pas une idée très adéquate du réel. Au sommet de ces déformations, l'immigration, surestimée de 10 à 25 points partout: les Italiens estiment ainsi qu'il y aurait $30 \%$ d'immigrés chez eux, quand ils ne sont que $7 \%$; les Américains qu'il y en aurait $32 \%$, quand il y en a $13 \%$, et les Français qu'il y aurait $28 \%$, quand il y en a $10 \%$ ! Autre surestimation, le nombre de chômeurs: surestiméde 15 (Allemagne) à 37 points (Italie). Ou les personnes âgées: les Italiens s'imaginent qu'elles sont $48 \%$-contre $21 \%$ en réalité. Bref, la plupart des grandes nations s'imaginent qu'elles sont composées majoritairement d'immigrés, devieux et de chômeurs. L'identité religieuse fait également l'objet d'une totale méconnaissance: avec une surestimation généralisée du nombre de musulmans (voir ci-dessus) et une sousévaluation du nombre de chrétiens : en France, ils sont évalués à $40 \%$ (au lieu de $63 \%$ en réalité). Les Américains les évaluent à $56 \%$ (au lieu de $78 \%$ ). Les pays du Nord ou d'Asie n'échappent pas à la tendance, même si leur marge d'erreur est moins élevée.

L'enquête s'amuse à dresser un Index de l'ignorance où l'Italie et les États-Unis arrivent en tête, la France occupant la $6^{\mathrm{e}}$ place. C'est donc un défi qui est posélà aux politiques: doivent-ils s'évertuer à éduquer les concitoyens de sorte qu'ils se forgent une perception plus adéquate de la réalité, ou, prenant le parti de ces illusions, adapter leurs propositions à cette vision tronquée du réel? On imagine sans mal ce que cette " politique de l'ignorance » pourrait produire. 
Les résumés demandés sont plutôt courts également, pouvant même être limités à une seule phrase, comme celui ci-dessous, sachant qu'un résumé d'une phrase peut être demandé dans une épreuve de concours.

Une étude de l'institut Ipsos réalisée dans quatorze pays montre que les peuples n'ont pas une perception très adéquate de leurs sociétés, surestimant le pourcentage de chômeurs, d'immigrés, de séniors et de musulmans par rapport aux chrétiens, ce qui place les politiques devant une alternative : adapter leurs propositions à cette représentation déformée ou aider plutôt les concitoyens à la corriger?

\subsection{PRINCIPALES DIFFICULTÉS DES ÉTUDIANTS}

Relativement aux recommandations officielles citées plus haut, les imperfections relevées dans les résumés (ceux d'une phrase et ceux plus longs) consistent, premièrement, dans le fait que les étudiants ont tendance à se distancier trop du texte d'origine. Cela se manifeste, d'une part, par des formulations qui, sans forcément comporter des contresens, constituent des extrapolations, comme dans les exemples suivants :

- L'enquête... aborde un délicat problème...

- Les politiques sont responsables de ces résultats.

- L'enquête essaye de faire réagir les politiques.

D'autre part, les étudiants donnent souvent l'impression de ne pas pouvoir se retenir de ramener les différents faits relatés à eux, ce qui passe par des marques explicites de la subjectivité, telles que le pronom nous ou le déterminant notre, comme dans les exemples suivants :

- Les résultats nous montrent que...

- Nous pouvons constater que notre perception de la société est illusoire.

- Un défi important se pose à chacun de nous.

Deuxièmement, concernant l'utilisation de "son propre langage », elle donne souvent lieu à l'emploi de termes inappropriés, comme dans les exemples cidessous.

- L'enquête révèle la perception que les concitoyens [au lieu de citoyens] se font de leurs pays [au lieu de sociétés].

- Les politiques devraient donc éduquer les citoyens pour qu'ils se forgent une situation [au lieu de vision, représentation, idée] plus adéquate de la réalité.

- Afin de comprendre le détachement [au lieu de décalage ou différence] entre la vision des citoyens du monde et la réalité sociale...

Même sans être clairement inadéquats au contexte, certains choix lexicaux modifient le point de vue par rapport à celui exprimé dans le texte d'origine, comme nous le montrerons plus bas dans l'illustration de la démarche. L'une des souscompétences lexico-discursives à travailler est donc celle qui permet de saisir les 
nuances sémantiques différenciant des expressions «concurrentes» en termes de point de vue et d'orientation de l'attention ou de focalisation. Les choix lexicaux inappropriés faits dans les exemples cités ci-dessus peuvent eux aussi être analysés de cette façon. Ainsi, en écrivant concitoyens, le locuteur oriente, à travers le préfixe con-, l'attention sur le sujet qui y réfère : ici, l'enquête et donc « les enquêteurs », qui se trouvent de ce fait associés aux citoyens enquêtés comme étant de la même citoyenneté (ce qui ne peut pas être affirmé et ce qui n'est pas pertinent). En employant le terme situation, dans l'exemple suivant, le locuteur reformulant introduit un point de vue «objectivant», en orientant l'attention du lecteur sur la « réalité », au lieu d'adopter un point de vue «subjectivant», en orientant l'attention sur les sujets enquêtés et leurs idées sur la réalité. Enfin, le terme détachement, à travers le préfixe dé-, introduit dans la sphère focalisée un état préalable consistant dans un «attachement", ce qui n'est clairement pas approprié dans ce contexte, où l'on ne peut pas présupposer une préalable coïncidence entre la réalité et la vision de la réalité dont témoignent les enquêtés.

Après avoir analysé les difficultés des étudiants, notamment celles concernant le vocabulaire, la méthode que nous leur avons proposée consiste à diviser la tâche de résumer en trois sous-tâches successives : (1) réduction du texte, en éliminant les fragments «secondaires» (répétitions, illustrations, précisions complémentaires); (2) nouvelle réduction, en vue d'atteindre la longueur exigée (tel nombre de mots ; une seule phrase), en faisant attention à la cohésion du texte ; (3) nouvelle reformulation passant par l'emploi de parasynonymes adéquats, nécessitant parfois des réajustements morphosyntaxiques. C'est cette dernière étape qui, à travers la recherche et l'analyse comparative des parasynonymes, donne lieu à une réflexion sur les valeurs sémantico-pragmatiques d'expressions, avec le but de choisir des parasynonymes qui correspondent le mieux au contexte (c'est-à-dire au but communicatif et à l'orientation attentionnelle de l'énoncé et du texte) ou, du moins, qui s'éloignent le moins possible du sens de l'expression de départ, quitte, à défaut, à garder dans la version finale du résumé certaines expressions d'origine.

\section{ILLUSTRATION}

Nous allons illustrer à présent la phase 3 du résumé, telle que décrite dans la section précédente, à partir d'un résumé-phrase «fidèle » (élaboré lors de la phase 2), comme celui ci-dessous, choisi parmi les productions préalables réalisées par les étudiants individuellement (ou à deux), avec des modifications apportées collectivement.

Une enquête menée par l'institut Ipsos dans quatorze pays montre que les peuples n'ont pas une perception très adéquate de leurs sociétés, surestimant le nombre de chômeurs, d'immigrés, la place de l'islam par rapport au christianisme et le vieillissement de la 
population, ce qui pose un défi aux politiques : éduquer les concitoyens de sorte qu'ils se forgent une perception plus adéquate de la réalité ou adapter leurs propositions à cette vision tronquée du réel?

\section{1. ÉLABORATION DE PARAPHRASES EN PASSANT PAR LA RECHERCHE DE SYNONYMES}

Le but de la phase 3 du résumé est d'en élaborer une nouvelle version, en remplaçant, là où cela semble possible, les termes d'origine par des expressions synonymiques adaptées au contexte (ou parasynonymes), et en opérant d'éventuels réajustements syntaxiques ou morphosyntaxiques, tout en évitant les répétitions.

Après une discussion en petits groupes, chaque groupe s'occupant d'un fragment différent (ex. une enquête menée par l'institut Ipsos), sans cependant perdre de vue le contexte global, les étudiants font des propositions, qui sont ensuite compilées et visualisées à l'aide d'un tableau blanc interactif ou d'un vidéoprojecteur, comme celles ci-dessous, placées entre parenthèses et mises en évidence par les caractères gras.

Une enquête (une étude) menée (effectuée / réalisée / conduite) par l'institut Ipsos dans quatorze pays montre (indique / prouve) que les peuples (gens / citoyens) n'ont pas une perception (connaissance / vision) très adéquate de leurs sociétés (pays) surestimant le nombre (pourcentage) de chômeurs, d'immigrés, la place de l'islam par rapport au christianisme (de musulmans par rapport aux chrétiens) et le vieillissement de la population (et de séniors), ce qui pose un défi (dilemme) aux politiques (politiciens / dirigeants) (ce qui place les... devant une alternative) : éduquer les concitoyens de sorte qu'ils se forgent (aider les concitoyens à se forger / développer) une perception (vision / évaluation / un jugement) plus adéquat(e) (correcte / juste) de la réalité (de la société) ou adapter leurs propositions (programmes) à cette vision (représentation) tronquée (déformée) du réel (de la réalité)?

Lorsque plusieurs parasynonymes et/ou reformulations sont proposés, on peut décider de faire des choix en vue de ne retenir qu'une seule option, afin de restreindre l'ampleur de l'analyse. Par exemple, dans la première phrase, on peut décider de retenir le verbe réalisée comme alternative à menée, en écartant effectuée et conduite.

\subsection{ANALYSE DE QUELQUES ALTERNATIVES EN GUISE D’EXEMPLES}

À partir d'un tel ensemble de propositions de reformulation, les étudiants sont invités à les analyser en essayant de préciser les différences de sens en termes de focalisation, c'est-à-dire en se demandant sur quel aspect de la référence telle expression permet d'orienter l'attention du lecteur, par rapport à telle autre 
expression. Les étudiants sont encouragés à s'aider de définitions de dictionnaire, ainsi que de sources encyclopédiques ou autres (par exemple, dans le cas cité ici, pour s'informer sur les activités de l'institut Ipsos), afin d'étayer leurs hypothèses. Il est important de souligner qu'on ne vise pas à aboutir à une analyse précise et définitive de chaque expression envisagée et que, comme nous l'apprend notre expérience, on peut s'attendre à des analyses très différentes voire divergentes. $\mathrm{Ce}$ qui compte, en définitive, ce n'est pas tant le résultat de chaque analyse mais la réflexion collective autour du vocabulaire, en vue d'un résultat à peu près satisfaisant aux yeux de tous les participants. Le but ultime étant, nous le rappelons, de sensibiliser les étudiants à la dimension subjective/intersubjective, ou attentionnelle, du lexique et du discours.

Par exemple, si l'on envisage l'alternative enquêtelétude, chaque option étant compatible avec la référence en question (c'est-à-dire avec l'action de l'institut Ipsos décrite dans le texte et avec ses activités en général), on peut remarquer, en s'appuyant sur la définition suivante du nom enquête dans Le Grand Robert électronique : «étude d'une question (sociale, économique, politique) par le rassemblement des avis, des témoignages des intéressés », que ce nom focalise les sujets enquêtés, ce qui n'est pas le cas du nom étude, qui focalise plutôt l'objet étudié : «effort intellectuel orienté vers l'observation et l'intelligence des êtres, des choses, des faits ». Concernant la décision de retenir ou non l'option étude dans la paraphrase et dans le résumé final, elle pourra s'appuyer sur l'orientation attentionnelle (ou focalisation) du passage contenant le mot enquête dans le texte de départ, où l'on remarque la place importante qui est donnée aux enquêtés. Cependant, on s'aperçoit que la version du résumé obtenue à l'issue de la phase 2 (cf. plus haut), celle sur laquelle porte le travail à cette étape (phase 3 ), a vu cette place se réduire au profit des résultats (conformément à l'orientation globale du texte, qui privilégie en définitive les résultats de l'enquête), ce qui fait finalement du terme étude une très bonne alternative. En conséquence, étant donné cette orientation attentionnelle retenue dans le résumé, on pourra remplacer le verbe mener par réaliser ou effectuer, ces deux verbes orientant l'attention sur l'accomplissement (ou la réalité résultante ou l'effet), à la différence de mener, qui focalise plutôt l'enquête ou l'étude elle-même ainsi que l'agent, donc ici l'institut Ipsos (cf. la définition du Grand Robert électronique : « s'adonner, se livrer à une activité soutenue, organisée dans un but précis, et dont on a (plus ou moins) l'initiative »). À ce stade, et étant donné ces réflexions, on peut considérer que la tournure passive de la phrase exprimant l'agent après le verbe sous forme de complément d'agent (par l'institut Ipsos), lui conférant un degré assez important de focalisation dû à sa position «prédicative », n'est pas parfaitement cohérente avec les choix précédents, ce qui peut motiver l'expression de cette information en tant que complément du sujet: une étude de l'institut Ipsos réalisée dans quatorze pays. 
Pour donner un autre exemple, on peut se pencher sur les options proposées au mot peuples: gens, citoyens. En prenant en compte la mention antérieure de pays, puis la mention ultérieure de sociétés, et au vu de la définition du mot peuple fournie par Le Grand Robert électronique : «ensemble d'humains vivant en société, habitant un territoire défini (...)», le mot peuples apparaît comme étant parfaitement adapté au contexte immédiat. Le mot gens, quant à lui, semble contraster quelque peu avec la tonalité scientifique du texte, en défocalisant par ailleurs, du fait de son sens plus général, les liens avec société et pays. Quant au terme citoyen, il focalise un sens en lien avec le terme politique, lequel apparaît dans la dernière partie du texte et du résumé (où est également employé le terme concitoyens). Ainsi, il est préférable de garder le mot peuples plutôt que de le remplacer par l'un des parasynonymes envisagés.

Ayant esquissé la façon dont il est possible d'aborder les différences entre les expressions proposées en vue de paraphrases, nous allons, pour finir, nous pencher sur un choix pouvant être envisagé à la fin du résumé-phrase, concernant le « défi » qui se pose aux politiques. Le résumé «fidèle », obtenu à l'issue de la phase 2 de rédaction, respecte l'ordre des deux solutions évoquées dans le texte d'origine: (1) éduquer les concitoyens de sorte qu'ils se forgent une perception plus adéquate de la réalité ou (2) adapter leurs propositions à cette vision tronquée du réel. En prenant en compte le fait, reconnu par les linguistes s'intéressant aux moyens syntaxiques de la focalisation (ex. Nølke, 2006), que la position finale dans la phrase est la plus focalisante, placer en position finale la solution exprimée par la proposition adapter leurs propositions à cette vision tronquée du réel peut donner l'impression que l'auteur valorise ou privilégie cette solution-là. Or, le texte d'origine se termine par une mise en garde, indiquant un jugement négatif vis-à-vis de cette deuxième solution (à travers notamment l'expression politique de l'ignorance): On imagine sans mal ce que cette "politique de l'ignorance» pourrait produire. Ainsi, une bonne façon de garder la trace de cette intention communicative dans le résumé est l'inversion de l'ordre des solutions évoquées, en donnant «le dernier mot» à la solution consistant à éduquer les concitoyens, cette focalisation syntaxique pouvant être renforcée par un moyen lexical : l'ajout de l'adverbe plutôt (cf. sa définition dans Le Grand Robert électronique : « de préférence »).

Nous reprenons ci-dessous le résumé-phrase, cité déjà dans la section 3.1 plus haut, obtenu après l'analyse, lors de la phase 3 , des parasynonymes et reformulations proposés lors de la phase 2 , et après l'adoption de certaines de ces propositions.

Une étude de l'institut Ipsos réalisée dans quatorze pays montre que les peuples n'ont pas une perception très adéquate de leurs sociétés, surestimant le pourcentage de chômeurs, d'immigrés, de séniors, et de musulmans par rapport aux chrétiens, ce qui place les politiques devant une alternative : adapter leurs propositions à cette représentation déformée ou aider plutôt les concitoyens à la corriger? 


\section{ADAPTATION AU CONTEXTE DU FRANÇAIS LANGUE ÉTRANGÈRE}

Après avoir pratiqué, dans le cadre d'activités autour du résumé de texte, des exercices de reformulation avec des étudiants de première année de licence de Sciences du langage dans une université française, principalement de langue maternelle française, nous voudrions réfléchir à l'application de ce type d'exercices au contexte FLE, universitaire également.

Le public visé est celui de niveau assez avancé, correspondant au stade où l'apprenant accède aux textes authentiques et, selon les termes de Francis Debyser, «plonge alors dans le lexique, illimité et non structuré, comme dans une mer où il risque évidemment de se noyer » (Galisson, 1970, Avant-propos, p. 5).

Dans le contexte du FLE, la motivation derrière les tâches de reformulation concerne notamment le développement du volet lexical de la compétence discursive, sachant que la reformulation implique également des choix aux niveaux de la syntaxe et de la morphosyntaxe, qui contribuent, eux aussi, comme nous l'avons illustré plus haut pour le moyen lié à l'ordre des syntagmes et des propositions, à la construction de l'orientation attentionnelle et argumentative de l'énoncé. Ce type d'analyse peut être particulièrement intéressant pour les futurs traducteurs et interprètes, en leur permettant de se sensibiliser à la dimension «attentionnelle »des formes linguistiques (relevant de la nature subjective/intersubjective du sens). En outre, avec le résumé de textes, le travail sur des formes linguistiques s'inscrit dans une activité mobilisant des tâches et des compétences plus générales, à savoir la compréhension et l'expression écrites.

Cependant, il n'est pas nécessaire de faire précéder les exercices de paraphrase de la rédaction préalable de résumés; un résumé « fidèle » (cf. la phase 2) pouvant être proposé par l'enseignant. Néanmoins, si l'on veut tirer profit de «l'ordre méthodologique » préconisé par Bakhtine (cf. la section 2 plus haut), c'est-à-dire de la contextualisation discursive permettant de cerner le but communicatif «global» du texte ainsi que les buts plus « locaux » (déterminant l'orientation de l'attention ou la focalisation), une bonne familiarisation avec le texte d'origine nous semble nécessaire.

Une démarche semblable peut également être proposée dans le cadre d'exercices de traduction, où, au lieu de travailler sur des synonymes et reformulations dans la langue étrangère, on peut mener un travail sur des expressions-traductions dans la langue maternelle (relativement à la fois au texte source et au texte cible). On peut également envisager des activités combinant les deux, c'est-à-dire : (1) le travail sur des synonymes et reformulations en langue source, puis (2), à partir d'un résumé «fidèle », le travail sur des termes «candidats» de la langue cible, en vue de la traduction du résumé. 


\section{CONCLUSION}

Les difficultés rédactionnelles des étudiants de première année de licence de Sciences du langage, observées lors d'activités liées à la production de résumés, indiquent, selon nous, une insuffisante maîtrise de la dimension «attentionnelle » (et donc intersubjective) du lexique et du discours. Un rédacteur compétent a acquis cette maitrise inconsciemment, grâce à la pratique intensive du discours (lecture et écriture), et sans doute aussi grâce à un certain "goût des mots », le faisant régulièrement envisager et réfléchir sur des alternatives d'expression en fonction de son but communicatif et l'interprétation visée. Des exercices rendant ces aspects plus explicites et conscients, sans pour autant pouvoir remplacer le bénéfice de la pratique soutenue du discours, peuvent aider les étudiants à améliorer leur compétence lexico-discursive. Dans le cas des étudiants «non natifs », futurs spécialistes de la langue française (traducteurs, interprètes, enseignants), la méthode proposée ici, s'inscrivant naturellement dans l'approche analytique de la langue qui est propre à l'apprentissage d'une langue étrangère, et consistant dans l'analyse des valeurs «attentionnelles » d'expressions, peut aider à accélérer le développement de cette compétence. 


\section{BIBLIOGRAPHIE}

Bakhtine, M. (Volochinov, V. N.) (2006). Le marxisme et la philosophie du langage. Essai d'application de la méthode sociologique en linguistique. Paris : Les Éditions de Minuit (traduction par M. Yaguello, œuvre originale parue en 1929).

Bréal, M. (1897). Essai de sémantique : science des significations. Paris : Hachette.

Fuchs, C. (1994). Paraphrase et énonciation. Paris : Ophrys.

Galisson, R. (1970). L'apprentissage systématique du vocabulaire. Paris : Hachette/Larousse.

Gardiner, A. H. (1989). Langage et acte de langage. Aux sources de la pragmatique. Lille: Presses Universitaires de Lille (traduction par C. Douay, œuvre originale: The Theory of Speech and Language, Oxford: Clarendon Press, 1932).

Kerbrat-Orecchioni, C. (2009). L'énonciation, de la subjectivité dans le langage. Paris : Armand Colin (quatrième édition).

Krieg-Planque, A. (2012). Analyser les discours institutionnels. Paris : Armand Colin.

Le Ny, J.-F. (2005). Comment l'esprit produit du sens. Notions et résultats des sciences cognitives. Paris : Odile Jacob.

Longhi, J. (2008). Sens communs et dynamiques sémantiques : l'objet discursif INTERMITTENT. Langages, 170, 109-124.

Nølke, H. (2006). La focalisation : une approche énonciative. In H. Włodarczyk (réd.), La focalisation dans les langues (pp. 59-80). Paris : L'Harmattan.

Rastier, F. (1987). Sémantique interprétative. Paris : PUF.

Tomasello, M. (2014). A Natural History of Human Thinking. Cambridge, Massachusetts, London, England : Harvard University Press. 